\title{
A Relação Entre a Imunodepressão e o Desenvolvimento de Cancro Cutâneo
}

\section{The Relationship Between Immunodepression and the Development of Skin Cancer}

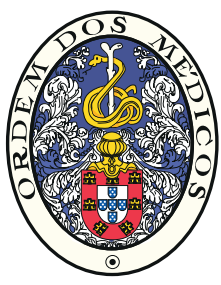

\author{
Maria Mendonça SANCHES ${ }^{1}$, Ana Rita TRAVASSOS ${ }^{1,2}$, Luís SOARES-DE-ALMEIDA $\rrbracket^{1,3,4}$ \\ Acta Med Port 2017 Jan;30(1):69-72 - http://dx.doi.org/10.20344/amp.7997
}

\section{RESUMO}

Os efeitos da imunodepressão no desenvolvimento de estados de doença têm constituído uma importante área de investigação, tendo sido verificadas associações entre a imunodepressão e o desenvolvimento de um vasto conjunto de comorbilidades, entre as quais se destacam as doenças infeciosas, doenças cardiovasculares e o cancro. A investigação dos efeitos da imunodepressão em humanos tem sido sobretudo realizada em indivíduos imunodeprimidos pelo vírus da imunodeficiência humana e indivíduos sob imunossupressão farmacológica após transplante, devido aos constrangimentos associados à obtenção de amostras de número relevante noutros contextos. De um modo geral, doentes imunodeprimidos tendem a apresentar maior incidência de neoplasias malignas. No que respeita aos doentes transplantados, o cancro cutâneo tem uma incidência significativamente aumentada, ao passo que, entre doentes portadores da infeção com vírus da imunodeficiência humana, apenas as neoplasias de origem infeciosa apresentam incidência aumentada. Este artigo apresenta uma revisão da literatura relativa aos efeitos da imunodepressão no desenvolvimento de tumores em humanos, com especial enfâse no desenvolvimento dos diferentes tipos de cancro cutâneo.

Palavras-chave: Imunossupressão/efeitos adversos; Imunossupressores; Neoplasias da Pele

\section{ABSTRACT}

The effects of immunodepression on several disease states have constituted an important area of research, leading to the identification of relevant associations between immunodepression and a vast set of comorbidities, including infectious diseases, cardiovascular diseases and cancer. Research on the effects of immunodepression has mostly been conducted in individuals under immunodepression by the human immunodeficiency virus and transplant recipients under pharmacological immunosuppression, due to the difficulties in obtaining relevant samples sizes in other contexts of immunodepression. Overall, immunosuppressed individuals tend to show increased incidence of malignancies, but only transplant recipients show significantly increased incidence of skin cancer; human immunodeficiency virus-infected individuals only show increased incidence of malignancies of infectious origin. This paper presents a literature review on the effects of immunodepression in the development of tumours in humans, with special emphasis on the development of the different types of skin cancer.

Keywords: Immunosuppression/adverse effects; Immunosuppressive Agents; Skin Neoplasms

\section{INTRODUCÃO}

A incidência de cancro cutâneo a nível mundial tem vindo a aumentar ao longo dos anos, sendo atualmente o cancro cutâneo, enquanto entidade única, a forma mais frequente de neoplasia maligna nos países desenvolvidos. ${ }^{1-4}$ A exposição a radiação ultravioleta (UV) está estabelecida como um dos principais fatores de risco para o desenvolvimento de lesões cutâneas, e em particular de neoplasias cutâneas. ${ }^{5-7}$ A radiação UV exerce o seu efeito cancerígeno na pele tanto por efeitos celulares diretos (nomeadamente alterações ao nível do DNA) como por alterações imunológicas que diminuem a vigilância celular com um aumento do potencial de transformação maligna. ${ }^{8}$

Os efeitos da imunodepressão no desenvolvimento de estados de doença têm constituído uma importante área de investigação. A imunodepressão está associada ao desenvolvimento de um vasto grupo de comorbilidades, entre as quais se destacam as doenças infeciosas, doenças cardiovasculares e o cancro. ${ }^{9,10}$ Estes efeitos da imunodepressão têm vindo a ser sobretudo estudados em doentes imunode- primidos pelo vírus da imunodeficiência humana $(\mathrm{VIH})$ e em doentes sob imunossupressão farmacológica após transplante, devido aos constrangimentos associados à obtenção de amostras de número relevante noutros contextos. Este artigo apresenta uma revisão da literatura relativa aos efeitos da imunodepressão no desenvolvimento de tumores em humanos, com especial enfâse no desenvolvimento dos diferentes tipos de cancro cutâneo.

\section{Doentes transplantados}

A utilização de agentes imunossupressores para prevenir a rejeição de tecidos transplantados aumenta o risco de desenvolvimento de cancro em comparação com a população geral. ${ }^{11}$ Dados de uma análise de 175342 transplantes de órgão sólido nos EUA indicam um rácio de incidência padronizado (SIR) de 2,10 - o que, comparado com a população geral, representa um aumento de risco de aproximadamente 719 casos por 100000 pessoa-anos. ${ }^{11}$ Verificou-se um risco significativamente aumentado para

1. Serviço de Dermatologia. Hospital de Santa Maria. Centro Hospitalar Lisboa Norte. Lisboa. Portugal.

2. Centro de Dermatologia. Hospital CUF Descobertas. Lisboa. Portugal.

3. Clinica Universitária de Dermatologia de Lisboa. Faculdade de Medicina. Universidade de Lisboa. Lisboa. Portugal.

4. Unidade de Investigação em Dermatologia. Instituto de Medicina Molecular. Lisboa. Portugal.

$\triangle$ Autor correspondente: Luís Soares-de-Almeida. lalmeida@medicina.ulisboa.pt

Recebido: 29 de junho de 2016 - Aceite: 14 de dezembro de 2016 | Copyright $\odot$ Ordem dos Médicos 2017 
desenvolvimento de 32 tipos de cancro, sendo que os seguintes tipos de cancro tiveram um risco maior cinco vezes superior face à população geral: sarcoma de Kaposi (SIR $61,5)$, neoplasias cutâneas (não melanoma e melanoma SIR 13,9), linfoma não-Hodgkin (SIR 7,5), neoplasias do fígado (SIR 11,6), do ânus (SIR 5,8), da vulva (SIR 7,6) e do lábio (SIR 16,8). ${ }^{11}$

Diversos fatores etiopatogénicos têm sido propostos para explicar a incidência aumentada de cancro em indivíduos transplantados, incluindo a imunossupressão (modulada pela duração e intensidade de tratamento), a exposição solar (sobretudo no âmbito do desenvolvimento de cancro cutâneo), a presença de infeção concomitante, a diálise pré-transplante renal e a possibilidade de neoplasia transplantada a partir do dador. ${ }^{9}$ Estes fatores apresentam, no entanto, influências extremamente variáveis dependendo tipo de cancro e do caso clínico em concreto.

\section{Impacto no cancro cutâneo}

A pele é o órgão no qual mais frequentemente se desenvolvem neoplasias em indivíduos transplantados. ${ }^{10}$ As neoplasias malignas cutâneas representam cerca de $40 \%$ dos tumores malignos nesta população, sendo desenvolvidos em mais de $50 \%$ dos indivíduos. ${ }^{12,13}$ Os tipos de neoplasias cutâneas mais frequentemente reportadas em indivíduos transplantados são o carcinoma espinocelular (CEC), o carcinoma basocelular (CBC), o melanoma (MM) e o sarcoma de Kaposi (SK). ${ }^{14}$ Contrariamente à população geral, o CEC é mais frequente que o CBC com proporções reportadas de até 5:1.14-16 Comparativamente com a população geral, os indivíduos transplantados têm um risco 65 - 250 vezes superior de desenvolver CEC e 10 - 16 vezes superior de desenvolver $\mathrm{CBC} ;{ }^{13}$ no âmbito do MM a evidência do risco aumentado é menos consistente, ainda assim vários estudos demonstram um risco aumentado em cerca de três vezes. ${ }^{11,17}$

Alguns dos fatores de risco reconhecidos para o desenvolvimento de cancro cutâneo na população em geral estão também implicados no desenvolvimento destas neoplasias em indivíduos transplantados, incluindo fotótipos baixos (fotótipos I - III), idade avançada, história de cancro cutâneo, queratoses actínicas, exposição solar crónica e exposição solar intermitente intensa com queimadura solar. ${ }^{18}$ Adicionalmente existem fatores de risco específicos dos indivíduos transplantados, incluindo a imunossupressão (sobretudo de elevada intensidade e duração), história de infeção pelo vírus do papiloma humano (HPV), idade avançada aquando do transplante, baixa contagem de linfócitos CD4 e tipo de órgão transplantado (o transplante cardíaco está associado a maior risco de cancro cutâneo, ${ }^{19}$ seguido do transplante renal, ${ }^{20}$ por sua vez seguido do transplante hepático). ${ }^{18,21}$

A exposição à radiação UV constitui um fator de risco para o desenvolvimento de cancro cutâneo nesta população. A radiação é tida como um carcinogénio completo, envolvido tanto na iniciação como promoção do processo de oncogénese. ${ }^{18}$ Para além do seu efeito mutagénico direto sobre as células da pele, a radiação UV apresenta também efeitos imunossupressores (locais e sistémicos) que facilitam a transformação maligna. ${ }^{18}$ Clinicamente os efeitos da radiação UV são facilmente reconhecíveis, não só pela elevada frequência e incidência de CEC em zonas foto-expostas mas também pela elevada ocorrência, num curto intervalo de tempo, de neoplasias cutâneas em doentes transplantados em zonas geográficas associadas a uma maior exposição solar. ${ }^{22}$

O grau de imunossupressão pós-transplante está correlacionado com o desenvolvimento de cancro cutâneo. ${ }^{23}$ Embora os mecanismos que originam um maior risco de cancro cutâneo em indivíduos transplantados não estejam completamente elucidados, tem sido proposto que o aumento do risco advém da combinação dos seguintes fatores: (1) vigilância imunológica reduzida, que facilita a sobrevivência e proliferação de células anómalas; (2) efeitos carcinogénicos diretos dos agentes imunossupressores, tais como a azatioprina e a ciclosporina e (3) proliferação de vírus oncogénicos, facilitada no contexto de imunossupressão. ${ }^{23}$ Do ponto de vista clínico, o impacto da imunossupressão é suportado por dados que indicam que a incidência de cancro cutâneo aumenta com a intensidade e duração do tratamento e, em sentido contrário, a incidência diminui após a cessação de terapêutica imunossupressora. ${ }^{24} \mathrm{O}$ impacto específico de diferentes agentes imunossupressores está ainda pouco explorado. Ainda assim, existem indicações de que a azatioprina e a ciclosporina podem influenciar o desenvolvimento de cancro cutâneo tanto por efeitos mutagénicos, como por efeitos imunossupressores. ${ }^{18}$ Efeitos mutagénicos têm sido detetados após exposição a radiação UVB e azatioprina, ${ }^{25,26}$ administração de inibidores da calcineurina, como a ciclosporina tem sido associada a alterações da morfologia celular, à inibição da função do gene p53 e à inibição da reparação de DNA (ligam-se às imunofilinas e inibem a síntese de IL-2 por bloqueio da transcrição do DNA), ${ }^{27-29}$ havendo assim evidência de que representam um maior risco de desenvolvimento de cancro cutâneo face a outros agentes imunossupressores. ${ }^{30}$

Tendo em conta o impacto do cancro cutâneo nos indivíduos transplantados, a implementação de estratégias de prevenção adequadas poderá desempenhar um papel fundamental na melhoria da qualidade de vida e na diminuição da morbilidade e mortalidade associadas..$^{31,32}$ Os comportamentos direcionados para a proteção da exposição solar são efetivos na redução do risco de cancro cutâneo e devem ser encorajados; estes incluem o uso de protetor solar, chapéus de aba larga e vestuário foto-protetor, assim como evitar a exposição em horas de pico de radiação UV..$^{9,33,34} \mathbf{A}$ estratégia de acompanhamento clínico deverá ser também adequada ao contexto de elevado risco de desenvolvimento de cancro cutâneo. Ulrich et al propõem um programa de seguimento composto por: (1) visita pré-transplante, para estratificação de risco, educação do doente e diagnóstico de eventuais lesões cutâneas e (2) seguimento de acordo com a estratificação de risco, por exemplo os doentes com baixo risco (sem história de lesões cutâneas, fotótipo 
alto ou sem outros fatores de risco) poderão ser seguidos anualmente, conforme o risco aumente, o seguimento deverá ser feito em intervalos mais curtos, podendo ser recomendado o seguimento mensal de doentes com risco muito elevado ( $p$. ex. apresentação melanoma maligno metastático). ${ }^{34}$ Adicionalmente, entre indivíduos transplantados, a utilização de estratégias de quimioprevenção primária ou secundária poderão ser equacionadas. A utilização de acitretina ou baixas doses de capecitabina aparentam ser eficazes na redução da incidência de CEC e CBC nesta população. ${ }^{35,36}$ Contudo, a sua utilização permanece pouco consensual.

\section{Doentes imunodeprimidos (VIH/SIDA)}

Os indivíduos infetados pelo VIH apresentam, de um modo geral, maior propensão para desenvolver neoplasias. ${ }^{37,38}$ No entanto, os padrões de desenvolvimento de neoplasias entre indivíduos imunodeprimidos pelo VIH não podem ser dissociados dos efeitos da terapêutica antirretroviral (TARV). ${ }^{39}$ Antes da introdução da TARV, estes doentes apresentavam maioritariamente neoplasias características e relacionadas com a síndrome de imunodeficiência, tais como SK, linfoma não-Hodgkin e carcinoma do colo do útero. ${ }^{39,40}$ Após a introdução da TARV, a incidência destas neoplasias entre indivíduos imunodeprimidos pelo $\mathrm{VIH}$ diminuiu substancialmente, sendo que se começou a verificar um aumento da incidência de outros tipos de neoplasias em comparação com a população em geral. ${ }^{39,40}$ Esta incidência aumentada de neoplasias não características do VIH representa hoje umas das principais causa de mortalidade nos doentes infetados com $\mathrm{VIH} .{ }^{39,40}$

Os mecanismos pelos quais o $\mathrm{VIH}$ origina uma incidência aumentada de cancro não estão completamente elucidados, mas pensa-se que os seguintes fatores desempenhem um papel fundamental neste processo: (1) deterioração imunológica (i.e., imunodepressão); (2) efeitos diretos do VIH em processos celulares; (3) coinfecção por outros vírus oncogénicos; (4) fatores ambientais, tais como tabaco e radiação $U V^{41,42}$

\section{Impacto no cancro cutâneo}

No contexto do cancro cutâneo em concreto, não existe evidência de um risco de desenvolvimento de cancro cutâneo particularmente aumentado (com exceção do SK em doentes não tratados com TARV). ${ }^{38}$ Uma metanálise de sete estudos de grande dimensão conduzida por Grulich et al detetou uma incidência aumentada de cancro, de um modo geral, entre indivíduos imunodeprimidos pelo $\mathrm{VIH} .{ }^{38}$ No entanto, a incidência tende a estar significativamente aumentada apenas nas neoplasias de etiologia infeciosa nomeadamente associadas a determinados vírus, como por ex.: o vírus Epstein-Barr - VEB, o vírus do papiloma humano - HPV, o poliomavirus das células de Merkel - MCC e o herpes vírus humano tipo 8 - HHV8, embora também se tenha verificado nas neoplasias malignas do fígado e do estômago. ${ }^{38}$ Quanto ao HPV, sabemos que algumas estirpes estão associadas a neoplasias benignas (p.ex. papilomas e queratoacantomas) e malignas (p.ex. cancro cutâneo não melanoma), especialmente em doentes imunodeprimidos. No entanto, apesar de já dispormos de vacinação eficaz contra algumas das estirpes mais associadas ao cancro do colo do útero e desta já fazer parte do Plano Nacional de Vacinação, ${ }^{43}$ esta não protege contra as estirpes HPV com maior tropismo cutâneo. Nos últimos anos vários estudos mostraram que, em modelos animais (incluindo em condições de imunossupressão) e mesmo em condições de infecção já estabelecida, a vacinação contra as estirpes cutâneas do HPV é possível e eficaz na prevenção destas neoplasias, tanto benignas como malignas. ${ }^{44,45}$

Por outro lado, na maioria dos tumores epiteliais, e em especial no cancro cutâneo não-melanoma, não se verificaram taxas de incidência significativamente aumentadas face à população em geral. ${ }^{38}$ Estes dados são indicativos de que a imunodepressão pelo VIH tem um papel limitado no desenvolvimento de cancro cutâneo, ao contrário do que se verifica nos indivíduos transplantados.

\section{CONCLUSÕES}

Doentes imunodeprimidos tendem a apresentar, de modo geral, maior incidência de neoplasias malignas. No que respeita aos doentes transplantados, o cancro cutâneo tem uma incidência significativamente aumentada, ao passo que entre doentes com infeção $\mathrm{VIH}$ apenas as neoplasias com origem infeciosa apresentam incidência aumentada. A abordagem clínica e o acompanhamento de doentes transplantados deverá ser estratificado e individualizado de forma a refletir o risco particularmente aumentado de desenvolvimento de cancro cutâneo nesta subpopulação.

\section{AGRADECIMENTOS}

$O$ apoio editorial e medical writing foi realizado pela ARC BIOSCIENCES - Scientific Consulting, Lda (ARC Publishing), com patrocínio da LEO Farmacêuticos.

\section{CONFLITOS DE INTERESSE}

Luís Soares-de-Almeida foi preletor em vários simpósios patrocinados pela LEO Farmacêuticos. Não há conflitos de interesse financeiros ou pessoais correlacionados com esta publicação.

\section{REFERÊNCIAS}

1. American Cancer Society. What are the key statistics about basal and squamous cell skin cancers? Atlanta: American Cancer Society; 2016.

2. Mohan SV, Chang AL. Advanced basal cell carcinoma: epidemiology and therapeutic Innovations. Curr Dermatol Rep. 2014;3:40-5.

3. Gandhi SA, Kampp J. Skin cancer epidemiology, detection, and management. Med Clin North Am. 2015;99:1323-35.
4. Siegel R, Naishadham D, Jemal A. Cancer statistics, 2013. CA Cancer J Clin. 2013;63:11-30.

5. Diepgen TL, Mahler V. The epidemiology of skin cancer. Br J Dermatol. 2002;146:1-6

6. Miller SJ. Biology of basal cell carcinoma (Part I). J Am Acad Dermatol 1991;24:1-13. 
7. Marzuka AG, Book SE. Basal cell carcinoma: pathogenesis, epidemiology, clinical features, diagnosis, histopathology, and management. Yale J Biol Med. 2015;88:167-79.

8. Narayanan DL, Saladi RN, Fox JL. Ultraviolet radiation and skin cancer. Int J Dermatol. 2010;49:978-86.

9. Chapman JR, Webster AC, Wong G. Cancer in the transplant recipient. Cold Spring Harb Perspect Med. 2013;3:pii:a015677.

10. Vajdic CM, van Leeuwen MT. Cancer incidence and risk factors after solid organ transplantation. Int J Cancer. 2009;125:1747-54.

11. Engels EA, Pfeiffer RM, Fraumeni JF, Kasiske BL, Israni AK, Snyder $\mathrm{JJ}$, et al. Spectrum of cancer risk among US solid organ transplant recipients. JAMA. 2011;306:1891-901.

12. Euvrard S, Kanitakis J, Claudy A. Skin cancers after organ transplantation. N Engl J Med. 2003;348:1681-91.

13. Greenberg JN, Zwald FO. Management of skin cancer in solid-organ transplant recipients: a multidisciplinary approach. Dermatol Clin. 2011;29:231-41.

14. Chockalingam R, Downing C, Tyring SK. Cutaneous squamous cell carcinomas in organ transplant recipients. J Clin Med. 2015;4:1229-39.

15. Caforio AL, Fortina AB, Piaserico S, Alaibac M, Tona F, Feltrin G, et al. Skin cancer in heart transplant recipients: risk factor analysis and relevance of immunosuppressive therapy. Circulation. 2000;102:222-7.

16. Ramsay HM, Fryer AA, Hawley CM, Smith AG, Harden PN. Nonmelanoma skin cancer risk in the Queensland renal transplant population. Br J Dermatol. 2002;147:950-6.

17. Mühleisen B, Petrov I, Frigerio S, Dziunycz P, French LE, Hofbauer GFL. Pronounced allelic imbalance at D9S162 in skin squamous cell carcinoma of organ transplant recipients. Arch Dermatol. 2012;148:697703.

18. Kovach BT, Stasko T. Skin cancer after transplantation. Transplant Rev. 2009;23:178-89.

19. Fortina AB, Caforio AL, Piaserico S, Alaibac M, Tona F, Feltrin G, et al. Skin cancer in heart transplant recipients: frequency and risk factor analysis. J Heart Lung Transplant. 2000;19:249-55.

20. Kasiske BL, Snyder JJ, Gilbertson DT, Wang C. Cancer after kidney transplantation in the United States. Am J Transplant. 2004;4:905-13.

21. Frezza EE, Fung JJ, van Thiel DH. Non-lymphoid cancer after liver transplantation. Hepatogastroenterology. 1997;44:1172-81.

22. Bavinck JN, De Boer A, Vermeer BJ, Hartevelt MM, van der Woude FJ, Claas $\mathrm{FH}$, et al. Sunlight, keratotic skin lesions and skin cancer in renal transplant recipients. Br J Dermatol. 1993;129:242-9.

23. Athar M, Walsh SB, Kopelovich L, Elmets CA. Pathogenesis of nonmelanoma skin cancers in organ transplant recipients. Arch Biochem Biophys. 2011;508:159-63.

24. Otley CC, Coldiron BM, Stasko T, Goldman GD. Decreased skin cancer after cessation of therapy with transplant-associated immunosuppressants. Arch Dermatol. 2001;137:459-63.

25. O'Donovan P, Perrett CM, Zhang X, Montaner B, Xu Y-Z, Harwood CA, et al. Azathioprine and UVA light generate mutagenic oxidative DNA damage. Science. 2005;309:1871-4.

26. Ren $X, X u Y-Z$, Karran P. Photo-oxidation of 6-thioguanine by UVA: the formation of addition products with low molecular weight thiol compounds. Photochem Photobiol. 2010;86:1038-45.

27. Yarosh DB, Pena A V, Nay SL, Canning MT, Brown DA. Calcineurin inhibitors decrease DNA repair and apoptosis in human keratinocytes following ultraviolet B irradiation. J Invest Dermatol. 2005;125:1020-5.

28. Norman KG, Canter JA, Shi M, Milne GL, Morrow JD, Sligh JE.
Cyclosporine A suppresses keratinocyte cell death through MPTP inhibition in a model for skin cancer in organ transplant recipients. Mitochondrion. 2010;10:94-101.

29. Wu X, Nguyen BC, Dziunycz P, Chang S, Brooks Y, Lefort K, et al. Opposing roles for calcineurin and ATF3 in squamous skin cancer. Nature. 2010;465:368-72.

30. Wheless L, Jacks S, Mooneyham Potter KA, Leach BC, Cook J. Skin cancer in organ transplant recipients: more than the immune system. J Am Acad Dermatol. 2014;71:359-65.

31. Ulrich C, Kanitakis J, Stockfleth E, Euvrard S. Skin cancer in organ transplant recipients--where do we stand today? Am J Transplant. 2008;8:2192-8.

32. Zwald FO, Brown M. Skin cancer in solid organ transplant recipients: advances in therapy and management: part I. Epidemiology of skin cancer in solid organ transplant recipients. J Am Acad Dermatol. 2011;65:253-61.

33. Hofbauer GF, Anliker M, Arnold A, Binet I, Hunger R, Kempf W, et al. Swiss clinical practice guidelines for skin cancer in organ transplant recipients. Swiss Med Wkly. 2009;139:407-15.

34. Ulrich C, Jürgensen JS, Degen A, Hackethal M, Ulrich M, Patel MJ, et al. Prevention of non-melanoma skin cancer in organ transplant patients by regular use of a sunscreen: a 24 months, prospective, case-control study. Br J Dermatol. 2009;161:78-84.

35. Hardin J, Mydlarski PR. Systemic retinoids: chemoprevention of skin cancer in transplant recipients. Skin Therapy Lett. 2010;15:1-4.

36. Endrizzi B, Ahmed RL, Ray T, Dudek A, Lee P. Capecitabine to reduce nonmelanoma skin carcinoma burden in solid organ transplant recipients. Dermatol Surg. 2013;39:634-45.

37. Rubinstein PG, Aboulafia DM, Zloza A. Malignancies in HIVIAIDS: from epidemiology to therapeutic challenges. AIDS. 2014;28:453-65.

38. Grulich $A E$, van Leeuwen MT, Falster MO, Vajdic CM. Incidence of cancers in people with HIVIAIDS compared with immunosuppressed transplant recipients: a meta-analysis. Lancet. 2007;370:59-67.

39. Franceschi S, Lise M, Clifford GM, Rickenbach M, Levi F, Maspoli M, et al. Changing patterns of cancer incidence in the early - and late - HAART periods: the Swiss HIV Cohort Study. Br J Cancer. 2010;103:416-22.

40. Herida M, Mary-Krause M, Kaphan R, Cadranel J, Poizot-Martin I, Rabaud C, et al. Incidence of non-AIDS-defining cancers before and during the highly active antiretroviral therapy era in a cohort of human immunodeficiency virus-infected patients. J Clin Oncol. 2003;21:344753.

41. Carbone A, Gloghini A. AIDS-related lymphomas: from pathogenesis to pathology. Br J Haematol. 2005;130:662-70.

42. Deeken JF, Tjen-A-Looi A, Rudek MA, Okuliar C, Young M, Little RF, et al. The rising challenge of non-AIDS-defining cancers in HIV-infected patients. Clin Infect Dis. 2012;55:1228-35.

43. Direcção Geral da Saúde. Programa Nacional de Vacinação - Alteração do esquema da vacina contra infecções por vírus do Papiloma humano (HPV). Norma $n^{\circ}$ 016/2014 de 29/09/2014 atualizada a 01/10/2014. Lisboa: DGS; 2014.

44. Vinzón SE, Braspenning-Wesch I, Müller M, Geissler EK, Nindl I, Gröne $\mathrm{HJ}$, et al. Protective vaccination against papillomavirus-induced skin tumors under immunocompetent and immunosuppressive conditions: a preclinical study using a natural outbred animal model. PLoS Pathog. 2014;10:e1003924.

45. Vinzón SE, Rösl F. HPV vaccination for prevention of skin cancer. Hum Vaccin Immunother. 2015;11:353-7. 